\title{
Gradhiva
}

GRADHIV

Revue d'anthropologie et d'histoire des arts

$7 \mid 2008$

Le possédé spectaculaire

\section{L'exposition Animal au musée Dapper : entretien avec Christiane Falgayrettes-Leveau, directrice}

\section{CpenEdition}

Journals

Édition électronique

URL : http://journals.openedition.org/gradhiva/1123

DOI : 10.4000/gradhiva.1123

ISSN : 1760-849X

Éditeur

Musée du quai Branly Jacques Chirac

Édition imprimée

Date de publication : 15 mai 2008

Pagination : 144-147

ISBN : 978-2-915133-86-8

ISSN : 0764-8928

Référence électronique

«L'exposition Animal au musée Dapper : entretien avec Christiane Falgayrettes-Leveau, directrice », Gradhiva [En ligne], 7 | 2008, mis en ligne le 10 décembre 2008, consulté le 15 septembre 2020. URL http://journals.openedition.org/gradhiva/1123

(c) musée du quai Branly 


\section{L'exposition Animal au musée Dapper Entretien avec Christiane Falgayrettes-Leveau*, directrice}

Gradhiva : La première question que nous souhaiterions vous poser porte sur la genèse, l'origine de l'exposition Animal. Comment êtes-vous arrivée à choisir ce thème et en quoi est-ce original par rapport à ce qui a déjà été fait au musée?

CFL: La genèse est un moment difficile, quelles que soient les expositions; je ne sais pas trop comment ça arrive. Je travaille sur plusieurs projets, plusieurs thématiques, il y en a qui avancent plus vite que d'autres. Animal était peut-être un thème un peu plus facile comparé aux expositions que je faisais depuis un certain temps. II s'agit pour le musée non pas seulement de se mettre à la portée du public, mais d'essayer aussi d'avoir de temps en temps des thématiques qui se rapprochent davantage des sujets qui l'intéressent. Cela fait vingt ans que nous présentons des expositions et, pour Animal, je me suis posé la question des thèmes que je n'avais pas abordés et de ce qui pourrait bien répondre aux attentes du public, tout en restant quand même dans une thématique qui donne matière à réflexion. Je n'ai pas envie de faire des expositions uniquement pour séduire, ce n'est pas l'objectif du musée : son but est d'ouvrir, d'essayer d'apporter quelque chose, mais pas forcément de faire un travail de basse vulgarisation. Concernant Animal, il y a beaucoup de travaux d'anthropologie, notamment à travers le sacrifice. En revanche, il n'y a pas eu de grande exposition sur ce thème.

Gradhiva:Ya-t-il des pièces dont vous êtes particulièrement fière dans cette exposition et qui sont significatives du projet ou de l'orientation que vous souhaitiez lui donner?

CFL : II y a à peu près cent cinquante pièces; le travail de mise en regard des œuvres prend beaucoup de temps, entre

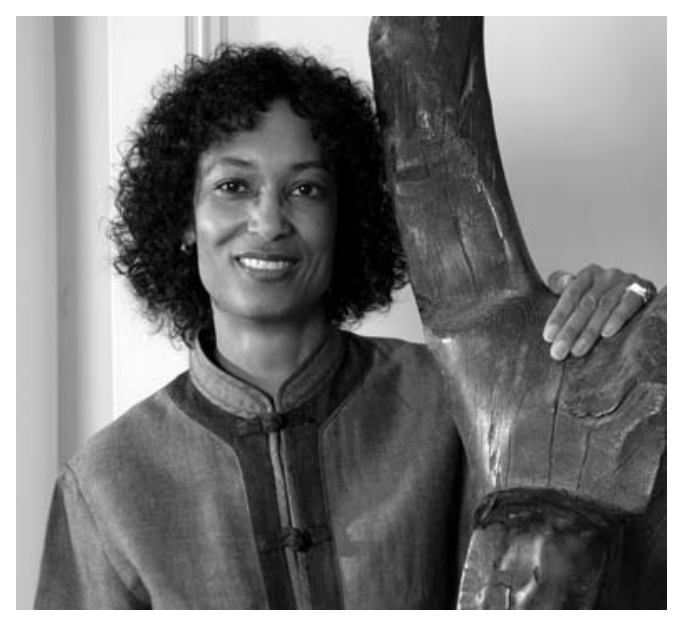

Portrait de Ch. Falgayrettes-Leveau. @ Photo Roland Poux. d'un tout petit objet yaka en bois de République démocratique du Congo (RDC), un objet de protection qu'on plaçait devant la maison des chasseurs. Cette pièce inédite se trouvait dans les réserves de Tervuren. II faut vraiment s'approcher pour voir les petites mâchoires accrochées autour de la statuette. A priori, ce n'est pas un objet esthétiquement important, mais il relève d'un processus plastique intéressant : Iorsqu'on regarde attentivement, on aperçoit des cornes d'antilope, qui ressemblent aux jambes d'un humain, positionnées à l'envers; de plus, ces mâchoires forment une accumulation qui donne une forte présence à l'objet. L'important est donc de rassembler des pièces pertinentes et qui restent à découvrir.

Gradhiva : Il y a une ligne thématique qui apparaît dans l'exposition, c'est la question des combinaisons entre homme, animal et esprit. De ce fait, il s'agit plus que d'une exposition sur l'animal, sur les transformations de l'animal... sur l'animal comme motif plastique décliné en formes très variées en fonction des cultures. Nous avons été particulièrement frappés par la juxtaposition de deux masques d'éléphant du Cameroun, l'un en bois, l'autre en perles. deux et trois ans. C'est très important d'obtenir des pièces rares, soit dans leur domaine, soit dans les musées, et parce qu'elles font partie des «trésors » qu'un musée n'a pas trop envie de «lâcher ». Je ne me pose pas vraiment la question de la préférence de certaines œuvres. Certaines sont magnifiques et c'est important de les montrer. Nous présentons par exemple une coupe royale luba avec des personnages et deux varans, qui est un chef-d'œuvre du musée de Tervuren (musée royal de l'Afrique centrale). Lorsqu'on peut exposer des objets qui ont rarement été vus par rapport à leur catégorie, on se dit qu'on a apporté quelque chose. Il y a une autre pièce de ce musée qui n'est pas nécessairement d'une grande beauté : il s'agit l'ouvrage. Je craignais de passer à côté de son approche. Nos travaux et nos discussions ont permis de ne pas oublier cette dimension dans l'exposition, qui est visuelle même si on met des panneaux avec du texte - tous les visiteurs ne les lisent pas. L'exposition rappelle ainsi qu'il s'agit d'une triade, l'homme, l'animal et puis l'esprit ou la présence du surnaturel; je crois que cette idée passe très bien pour le public. Nous avons peut-être apporté cette dimension à une exposition à propos de laquelle on nous disait au départ que le sujet était facile, qu'on allait simplement y voir des représentations d'ani-

* Entretien réalisé le $1^{\text {er }}$ février 2008. 


\section{Chronióue Scientifióue}

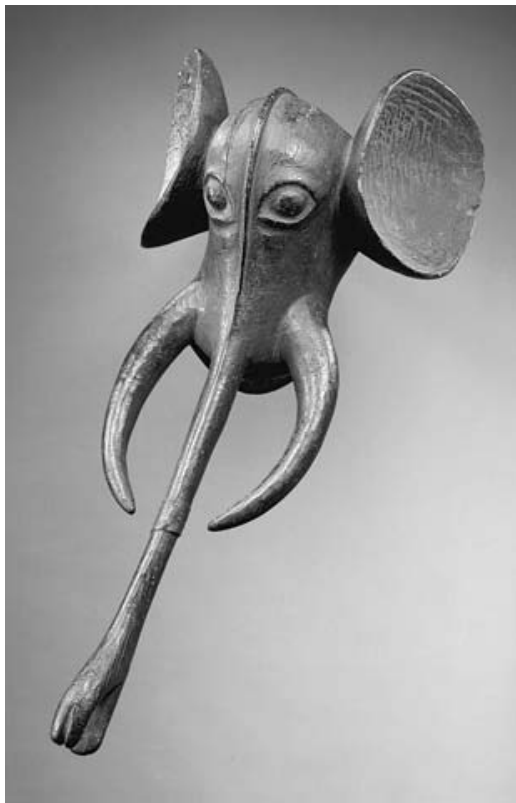

Fig. 5 Chefferie de Babanki-Tungo?, Cameroun, province du Nord-Ouest (département de Ngo-Ketunjia?), masque éléphant $n$ tshen, collection particulière. @ Archives Musée Dapper et Hugues Dubois.

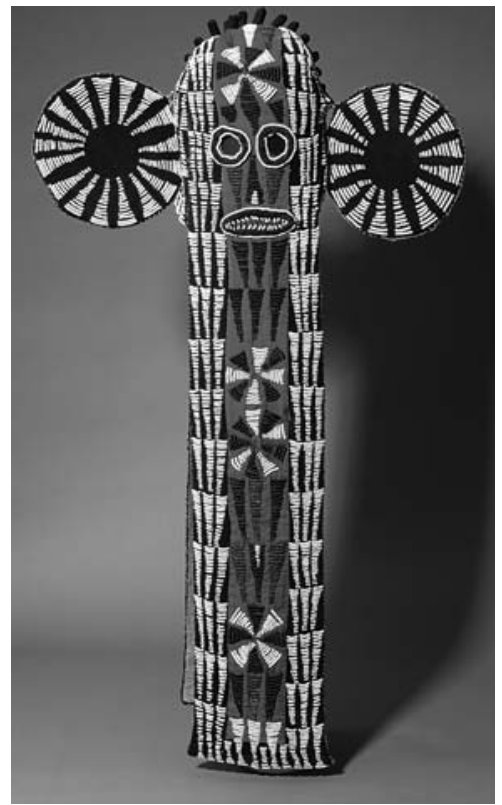

Fig. 6 Bamileke, Cameroun, province de l'Ouest, masque éléphant porté par les membres des associations kemjyeh et kwo'si. @ Sttatliches Museum für Volkerkunde, Munich, photo F. Autrum-Mulzer. car elle remet en cause le fait que des animaux soient représentés simplement parce qu'ils étaient chassés. C'est un peu la même orientation avec l'article sur la divination, abordée par Luc Pecquet, puisque nous n'avons pas de représentation dans l'exposition.

Gradhiva : II s'agit d'un article du catalogue sur la divination dans le sable par le renard, avec des photographies exceptionnelles.

CFL : Luc Pecquet est arrivé tout à fait par hasard, par l'intermédiaire de Daniela Bognolo qui a écrit sur le Burkina Faso. Elle m'a apporté des documents réalisés par Luc Pecquet représentant les traces dans le sable, qui m'ont beaucoup intriguée. Évidemmentc Pecquet a voulu se détacher de la divination par le renard au Mali même si nous voulions le ramener dans cette direction, certes très balisée, mais tout de même fort importante. II explique donc que les devins yela passaient leur temps à décrypter ces tables, à en discuter entre eux une vieille détermination qui ne correspondait pas vraiment à la fonction de la pièce. De temps en temps, on voit circuler des définitions, on les véhicule sans se poser de question... Puis arrive un moment où une appellation et un usage sont mis en question. En regardant de plus près ces pièces kuyu, en particulier cette relation, cette fusion entre l'humain et l'animal, on observe que les deux visages du singe et du personnage sur la tête duquel il est posé sont traités de la même façon : l'un et l'autre ont des dents taillées en pointe. On a un singe mais peu de données sur sa présence, alors que, a priori, la plupart de ces groupes sont liés au léopard. On a des données qui s'entrecroisent et qui ne sont pas si simples à interpréter. Souvent, on ne sait même pas de quel animal il s'agit, ce n'est pas une chimère, on est entre plusieurs figurations... Mais c'est parfois difficile d'aller plus loin. Et on ne peut pas réduire les systèmes de pensée en Afrique à des concepts très simples, en disant que c'est forcément tel animal.

Gradhiva : Pour le catalogue, vous avez fait appel aussi à un préhistorien, John Parkington. C'est l'une des premières fois que cela arrive?

CFL : Oui, c'est la première fois. Nous avons ciblé une région, l'Afrique du Sud, où la «tendance animale » est là depuis toujours. On ne pouvait pas présenter ce type d'images dans l'exposition, mais il s'agissait de demander à l'auteur d'interroger les peintures rupestres par rapport à notre thématique. Cette contribution est intéressante

2. Voir dans ce numéro la recension à propos de : Jérôme Souty, Pierre Fatumbi Verger. Du regard détaché à la connaissance initiatique. Paris, Maisonneuve et Larose, 200?. d'ailleurs lorsqu'ils n'avaient pas de clients; ils réfléchissaient aux systèmes graphiques. Et quand un client se rendait chez le devin, ce dernier avait obligation de dire s'il voyait quelque chose, même s'il devinait que cette personne allait mourir. Donc, c'est une relation complexe et tragique. Luc Pecquet travaillait initialement sur l'espace et l'environnement, et c'est à cette occasion qu'il a découvert ce système divinatoire. Je pense que son œil et sa réflexion par rapport à l'espace sont intéressants.

Gradhiva : Il y a aussi dans ce catalogue un article de Jérôme Souty, spécialiste du Brésil et de Pierre Verger, du candomblé de Salvador de Bahia en particulier. En revanche, dans l'exposition, l'Amérique noire est absente.

CFL : C'était compliqué. J'avais découvert Jérôme Souty un peu par hasard. J'ai trouvé son approche du travail de photographie de Verger ${ }^{2}$ passionnante et je lui ai demandé au cours d'une discussion si cela l'intéresserait de contribuer à l'ouvrage. On devait exposer les photographies de Pierre Verger dans l'une des salles, mais cela s'est révélé malheureusement trop difficile.

Gradhiva : II y a un autre aspect de l'exposition qui est complémentaire et vraiment fascinant, c'est la salle consacrée à l'installation de pailles tressées et agrafées de Julie Bessard. Comment voyez-vous l'articulation ou la complémentarité de cette première salle avec le reste de l'exposition?

CFL : C'est vrai que cette salle 1 est, depuis quelques années, consacrée aux artistes contemporains qui n'ont pas encore une grande notoriété. On a parfois exposé des artistes connus, comme Ousmane 
Sow, mais de façon générale je laisse la place à quelques sculptures ou photographies d'artistes peu connus. Cette fois-ci, l'artiste, Julie Bessard, est originaire de la Martinique, tandis que d'habitude les plasticiens viennent plutôt d'Afrique. Lors d'une mission en Martinique pour la mairie de Fort-de-France, il y a trois ans, j'avais visité des ateliers d'artiste et j'avais vu le travail de Julie. C'était prometteur et je l'avais gardé en tête. Lors de la préparation de l'exposition, je ne voulais pas exposer un artiste qui faisait uniquement de la sculpture animalière ; d'une part, c'est trop facile, et d'autre part ça ne pouvait pas fonctionner en introduction de ces pièces d'art africain, il $y$ aurait eu un hiatus. Je trouvais plus vivant et plus intéressant de rester dans une installation conceptuelle. Avec ces formes, ces pailles qu'elle façonne, qu'elle contraint avec des agrafes, le travail de Julie est surprenant. Elle a d'ailleurs rajouté des éléments pendant l'installation. On la voyait assise à compter ses agrafes, cela semble relever du rituel... Une partie de la culture de cette artiste est occidentale - elle est Martiniquaise, métisse -, mais elle est quand même très ancrée dans la Caraïbe. II y a d'ailleurs dans l'ouvrage un texte très poétique de Patrick Chamoiseau.

J'ai eu une appréhension pour l'installation de Julie lorsque nous travaillions au montage de l'exposition. Quand vous choisissez des sculptures, il n'y a pas de surprise, c'est surtout une affaire de disposition. Mais quand vous laissez l'espace à un artiste, bien qu'un certain nombre de choses aient été déterminées au préalable, vous ne savez pas ce qui va se passer. On commence à mettre la lumière, le son... Il y a des choses qui apparaissent... Mais il faut que ça reste cohérent par rapport à l'image de Dapper et que le dialogue se crée avec les espaces suivants présentant les arts anciens. Au fil du montage de l'installation, on se demandait si ce travail allait «fonctionner » par rapport à ce qui se passait de l'autre côté. J'ai été très surprise et contente de voir le public s'arrêter, peut-être plus que pour d'autres artistes. Non pas pour traverser

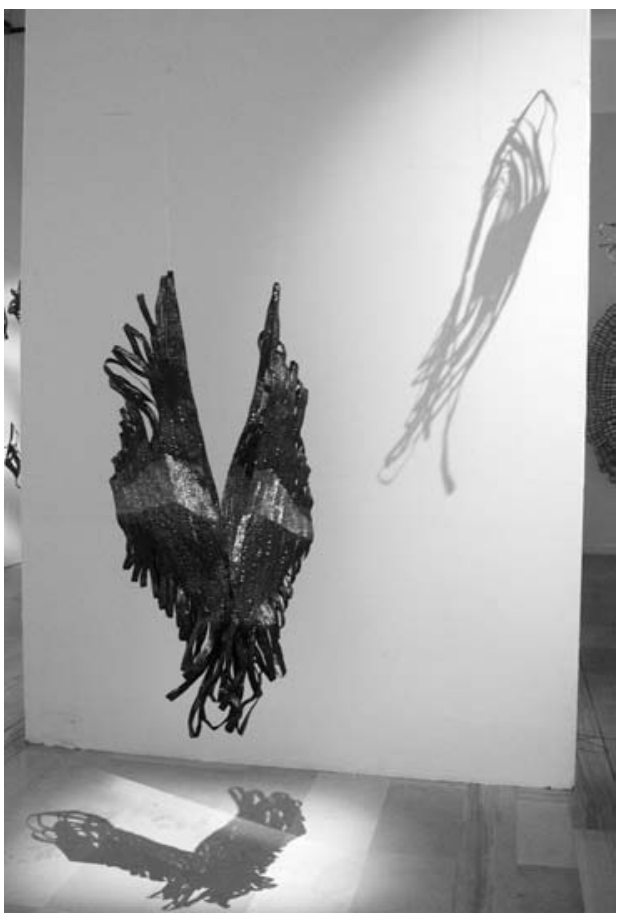

Fig. 7 Julie Bessard, Petites ailes, CMAC, Atrium, Martinique, 2005. @ Photo Robert Charlotte.
Présenter Julie Bessard non loin des sculptures d'Afrique, cela signifie que, même s'il y a modernité et créativité, il n'y a pas rupture totale entre ce que nous sommes aujourd'hui et les liens avec l'Afrique. Qu'elle soit repensée, détournée, tout ce qu'on veut, l'Afrique est là par certains cultes, par une forme de religiosité, de spiritualité, par la parole, par les contes... La présence de Chamoiseau apporte donc une dimension forte, qui est celle de la culture caribéenne, mais aussi la voix de l'un des intellectuels qui me semblent importants aujourd'hui, avec Édouard Glissant et quelques autres.

Gradhiva : Glissant avait déjà participé il y a quelques années au catalogue de l'exposition Lam métis.

CFL : Nos ouvrages constituent effectivement des suites, on commence avec certains, on continue avec d'autres et il y a des détours, parce que c'est bien - parce que nombre de visiteurs ne regardent pas à partir du moment où il s'agit d'art contemporain -, mais vraiment regarder.

Gradhiva : Le jeu de lumières, les fausses ombres, vraiment très réussies, suscitent une sorte de fascination. Julie Bessard a composé son installation après avoir vu le montage de l'exposition?

CFL : Non, c'était avant, elle avait simplement les plans. Elle avait déjà une partie des éléments et en a créé d'autres pour l'exposition. Au moment du montage, elle a enlevé puis remis des choses... On montait, nous, d'un côté, et elle était de l'autre, à son installation. dans ces espaces-là qu'il est intéressant de travailler. Et Patrick Chamoiseau s'intéresse de plus en plus à l'art contemporain, il écrit sur beaucoup d'artistes.

Gradhiva : Quels sont vos projets à venir?

CFL : Animal se termine le 20 juillet. À la rentrée, nous présentons l'exposition Femmes, au pluriel évidemment... Dans l'ouvrage, nous avons encore un texte d'Alfred Adler en introduction et on retrouvera deux ou trois auteurs comme Stefan Eisenhofer, mais nous avons aussi de nouveaux noms, Fatou Sow par exemple. 